Review

\title{
Preparation of Quantum Dot/Drug Nanoparticle Formulations for Tracea- ble Targeted Delivery and Therapy
}

\author{
Ken-Tye Yong ${ }^{1 凶}$, Yucheng Wang ${ }^{1}$, Indrajit Roy², Hu Rui ${ }^{1}$, Mark T. Swihart ${ }^{3}$, Wing-Cheung Law ${ }^{4}$, Sang Kyu \\ $\mathrm{Kwak}^{5}$, Ling Ye ${ }^{6}$, Jianwei Liư6, Supriya D. Mahajan7, and Jessica L. Reynolds ${ }^{7}$
}

1. School of Electrical and Electronic Engineering, Nanyang Technological University, Singapore 639798, Singapore

2. Department of Chemistry, University of Delhi, Delhi-110 007, India

3. Department of Chemical and Biological Engineering, University at Buffalo, The State University of New York, Buffalo, New York 14260-4200

4. Institute for Lasers, Photonics and Biophotonics (ILPB), The State University of New York at Buffalo, Buffalo, NY14260, United States

5. Division of Chemical and Biomolecular Engineering, School of Chemical and Biomedical Engineering, Nanyang Technological University, Singapore 637459

6. Institute of Gerontology and Geriatrics, Chinese PLA General Hospital, P. R. China

7. Department of Medicine, Division of Allergy, Immunology, and Rheumatology, University at Buffalo, The State University of New York, and Kaleida Health, Buffalo, New York, 14203, USA

Corresponding author: Dr. Ken-Tye Yong, School of Electrical \& Electronic Engineering, Nanyang Technological University, 639798, Singapore. Email: ktyong@ntu.edu.sg.

C Ivyspring International Publisher. This is an open-access article distributed under the terms of the Creative Commons License (http://creativecommons.org/ licenses/by-nc-nd/3.0/). Reproduction is permitted for personal, noncommercial use, provided that the article is in whole, unmodified, and properly cited.

Received: 2011.10.20; Accepted: 2011.12.04; Published: 2012.07.27

\begin{abstract}
Quantum dots (QDs) are luminescent nanocrystals with rich surface chemistry and unique optical properties that make them useful as probes or carriers for traceable targeted delivery and therapy applications. QDs can be functionalized to target specific cells or tissues by conjugating them with targeting ligands. Recent advancement in making biocompatible QD formulations has made these nanocrystals suitable for in vivo applications. This review provides an overview of the preparation of QDs and their use as probes or carriers for traceable, targeted therapy of diseases in vitro and in vivo. More specifically, recent advances in the integration of QDs with drug formulations for therapy and their potential toxicity in vitro and in vivo are highlighted. The current findings and challenges for optimizing QD/drug formulations with respect to optimal size and stability, short-term and long-term toxicity, and in vivo applications are described. Lastly, we attempt to predict key trends in QD/drug formulation development over the next few years and highlight areas of therapy where their use may provide breakthrough results in the near future.
\end{abstract}

Key words: Quantum dots, Drug Nanoparticle Formulations, Targeted Delivery

\section{Introduction}

A new approach to development of biocompatible nanoformulations that can target and treat human diseases involves the use of functionalized nanoparticles engineered to deliver drugs to the desired tissues or organs [1-2]. Although efficient internalization within cells and selective targeting are the most essential features of such a drug carrier, ideally it should also have long circulation time, self-regulation of drug 
release, low toxicity and minimal side effects [3-4]. In general, drug carriers consist of therapeutic agents within a nanoparticle system with characteristic size from 10 to $1000 \mathrm{~nm}$ [5]. Such a nanoparticle system will increase solubility and enhance stability of mostly poorly water soluble therapeutic agents, and will protect them from interacting with non-targeted cells and tissues, thus reducing toxicity [6].

Presently, two main categories of nanoparticles are widely employed for biological applications: inorganic nanoparticles and polymeric nanoparticles [7-9]. Polymeric nanoparticles can encapsulate drugs and release them in a regulated fashion through surface erosion of the nanoparticles, diffusion of the drug through the polymer matrix, or swelling followed by diffusion [10-12]. Alternatively, drug release can be triggered by changes in $\mathrm{pH}$ or temperature, the presence of an analyte such as enzyme, or external application of light or a magnetic field.[13-15] Over the last few decades controlled release polymeric nanoparticle technology has impacted virtually every branch of medicine, including oncology, ophthalmology, pulmonary medicine, orthopedics, immunology, neurology and dentistry [16-20]. However, traditional polymer particle formulations tend to be of relatively large size (200-500 $\mathrm{nm}$ ), and thus may not be ideal for specific applications, such as drug delivery to solid tumors and metastatic sites. The stability of these particles upon systemic administration may be compromised, and finally, these particles may lead to development of an immune response that may counteract therapy. Inorganic nanoparticles, on the other hand, can be made less than $20 \mathrm{~nm}$ in diameter, which not only makes them suitable for unimpeded circulation in the bloodstream and extravasation into tumor tissues, but may also permit excretion through renal filtration, without the need for biodegradation. Several such materials, including silica, gold nanoshells and gold nanoparticles are known for their low toxicity and biocompatibility for targeted imaging and therapy [21-23]. Many such materials, including quantum dots (QDs) and iron-oxide nanoparticles, have unique characteristics that enable their use as image contrast agents [24]. However, their rigid matrix does not generally allow for encapsulation and subsequent release of active molecules for conventional drug delivery purposes. Instead, drug molecules can be linked to, or combined with, the nanoparticles for targeted delivery purposes.

Bioconjugated quantum dots (QDs) show promise in applications spanning both diagnostics and therapeutics [25-27]. QDs have been used for more than a decade as optical contrast agents for bioimaging (see Figure 1) [28-31], and some comprehensive review articles have recently been published on the biological applications of QDs [32-33]. For targeted delivery applications, QDs must be conjugated with biorecognition ligands such as antibodies, DNA, biotin, streptavidin, or peptides [34-37]. For example, Gao et al. demonstrated the preparation of antibody-conjugated polymeric micelle coated CdSe/ZnS QDs for targeting and imaging of human prostate cancer growing in nude mice [38]. Cai et al. reported the in vivo targeting and imaging of tumor vasculature using arginine-glycine-aspartic acid (RGD) peptide-labeled CdTe/ZnS quantum dots (QDs) [39]. Yong et al. showed that bioconjugated CdSe/CdS/ZnS quantum rods and CdTe/CdS quantum dots can be used as ultrasensitive optical nanoprobes for tumor vasculature imaging in live animals [40-41]. More recently, Erogbogbo et al. demonstrated the preparation of peptide-conjugated phospholipid-micelle-encapsulated silicon quantum dots for in vivo tumor imaging without observing any toxicity at the cellular and tissue level (see Figure 1) [42-43]. All these studies have laid an important foundation in engineering of QDs for disease therapy research and applications. Recently, several research groups have demonstrated the integration of drug molecules with bioconjugated QDs for traceable drug delivery and therapy in vitro and in vivo [44-45]. Despite extremely active research on QDs and their application in bioimaging, the investigation of QD/drug nanoparticle formulations has only begun recently, due to the advancement of solution phase synthesis of QDs and bioconjugation chemistry for linking drug molecules to the QD surface. This review discusses the current status of this field, some of the critical challenges that remain, and measures that are being taken to overcome these challenges. 


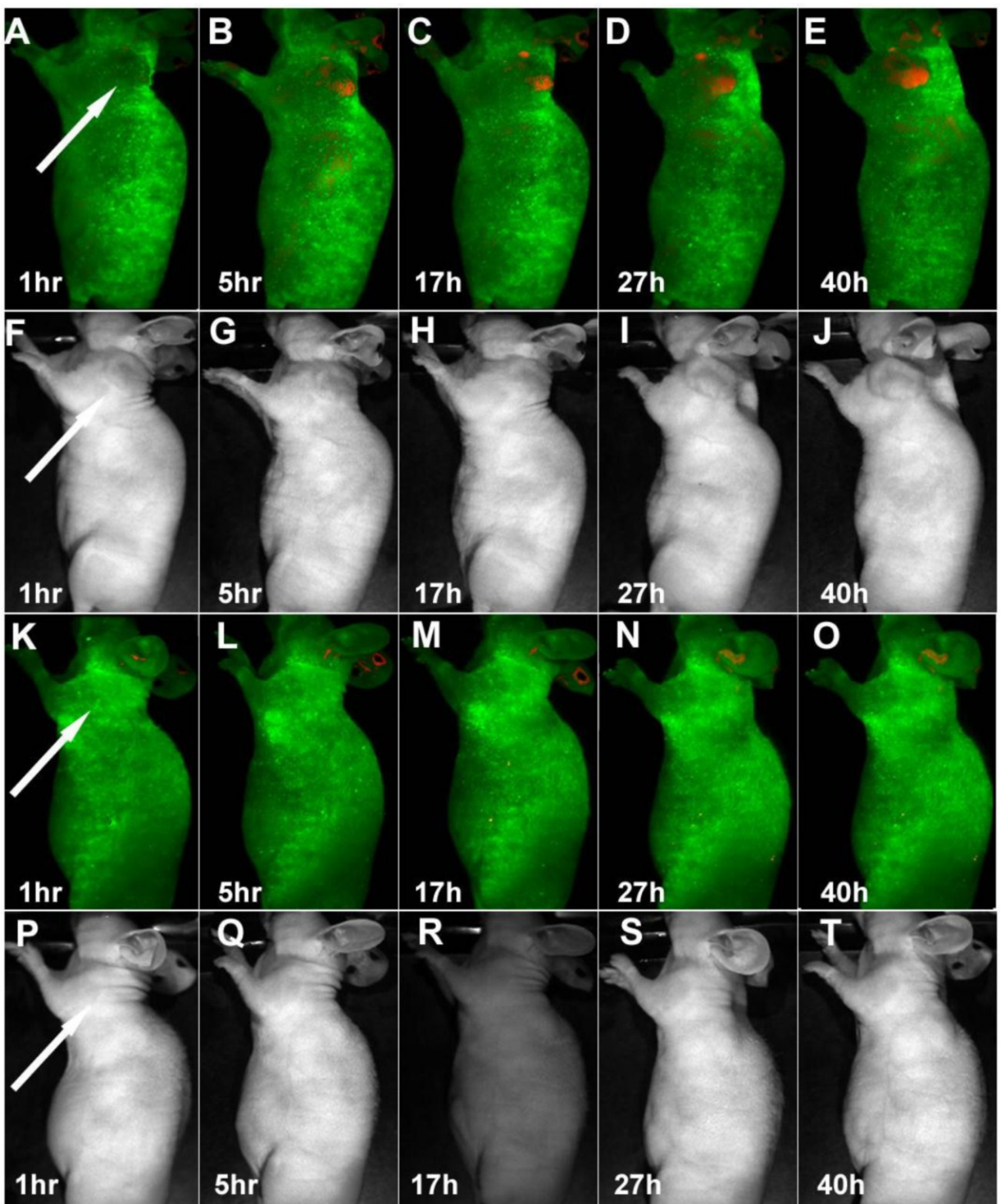

Figure I. Time-dependent in vivo luminescence imaging of Panc-I tumor bearing mice (left shoulder, indicated by white arrows) injected with silicon quantum dots conjugated with $(A-E)$ and without $(K-O) R G D$ peptide. All images were acquired under the same conditions. Autofluorescence and the unmixed SiQD signal are coded in green and red, respectively. Panels $\mathrm{F}-\mathrm{J}$ and panels $\mathrm{P}-\mathrm{T}$ correspond to the luminescence images in panels $A-E$ and $K-O$, respectively. Reprinted with permission from (Erogbogbo F, Yong KT, Roy I, Hu R, Law CW, Zhao W, Ding H, Wu F, Kumar R, Swihart MT, and Prasad PN et al. In Vivo Targeted Cancer Imaging, Sentinel Lymph Node Mapping and Multi-Channel Imaging with Biocompatible Silicon Nanocrystals. ACS Nano. 20I I; 5: 4I 3-423.). Copyright (20I I) American Chemical Society. 


\section{Synthesis, Characterization, and Optical Properties of Quantum Dots}

To date, colloidal core/shell QDs such as CdSe/ZnS, CdSe/CdS/ZnS, CdTe/CdSe and $\mathrm{InP} / \mathrm{ZnS}$ are commonly synthesized for biomedical applications [39, 46]. Among many approaches for making QDs, the hot colloidal synthesis approach remains the best method for making robust, high quality QDs [47-50]. This method was first described by Murray et al., who used pyrolysis chemistry of organometallic precursors containing cadmium and selenium to generate CdSe nanocrystals [51]. Later, this method was modified and continues to be a popular method for obtaining good quality of QDs [52]. For example, core CdSe QDs can be synthesized at high temperature (e.g. $300^{\circ} \mathrm{C}$ ) by the reaction between cadmium oxide dissolved in oleic acid and selenium dissolved in trioctylphosphine (TOPSe) [53]. This reaction results in the formation of monodispersed QDs [54]. To use QDs for biological applications, one must passivate the core QD with a thin layer of non-toxic high band gap material such as ZnS or ZnSe [55]. Such core/shell QDs have many advantages over unpassivated ones. For example, the chemical and optical stability of QDs can be maintained for longer periods of time when they are passivated with higher band-gap semiconductor materials [56]. The shell also significantly reduces the toxicity of the QDs, which makes them more suitable for biological applications. To date, many types of core/shell QDs were fabricated. However, only CdSe/ZnS, CdTe/ZnS, and CdSe/CdS/ZnS have been commonly used for in vitro and in vivo imaging [57]. Such core/shell QDs are created by epitaxially growing a higher band-gap semiconductor material around the core. Shelling CdSe QDs with ZnS results in small red-shifts $(\sim 10$ $\mathrm{nm}$ ) of the absorption and photoluminescence peaks of the QDs. In addition to cadmium-based QDs, many research teams are currently preparing cadmium-free QDs such as InP, CuInS 2 , and $\mathrm{Si}$, addressing concerns about CdSe and CdTe QD toxicity associated with the presence of cadmium. Compared to cadmium-based QDs, these QDs are less toxic and more promising for in vivo applications. However, production of high-quality QDs is more difficult for these materials than for Cd-based materials.
Most organic dyes display narrow absorption spectra and require specific excitation wavelengths to excite them [58-59]. In contrast, QDs have broad absorption spectra, allowing them to be excited by light of a wide range of wavelengths [60] (see Figure 2). This allows one to simultaneously excite QDs with different emission spectra for multiplex imaging using a single excitation source [61]. Organic dyes also have relatively broad emission spectra, resulting in the overlap of their fluorescence spectra, thus limiting their use for multiplex imaging [62]. In contrast, QDs have narrow emission spectra, which can be manipulated by changing the core size and composition of the QDs. More importantly, the QDs can be tuned to emit emission ranging from UV to near-infrared region. The high photostability of QDs is another unique feature from QDs for fluorescence imaging applications [63]. Unlike organic dyes, which may photobleach rapidly, QDs are stable and can withstand many cycles of excitation for long periods of time with a high level of brightness [64]. For example, dihydrolipoic acid-functionalized core/shell CdSe/ZnS QDs showed no change in the luminescence intensity after more than 10 hours of continuous excitation, and were 100 times as stable as rhodamine dye. In addition, QDs have a long luminescence lifetime after excitation and this can be an advantage for time-gated imaging. The fast fluorescence emission of organic dyes is similar to the short lifetime of the autofluorescence background from cells and tissues; thus, the signal-to-noise ratio is reduced. However, QDs generally emit light with a decay time of 30 to $100 \mathrm{~ns}$, which is much slower than that of the autofluorescence background decay, while remaining fast enough to maintain a high photon turnover rate. In time-gated analysis, photons detected in the first few ns after pulsed excitation are disregarded to decrease background noise and increase sensitivity. This advantage has been utilized to produce images of 3T3 mouse fibroblasts with a high signal-to-background ratio and to monitor the dynamics of erbB1 and erbB3 receptors $[2,33,65]$. In this case, this technique can be used to differentiate the erbB3 receptors labeled with citrine and erbB1 receptors labeled with QDs. Therefore, owing to their high brightness, photostability, and long decay time, the dynamics of QDs can be optically traced in vitro and in vivo. 


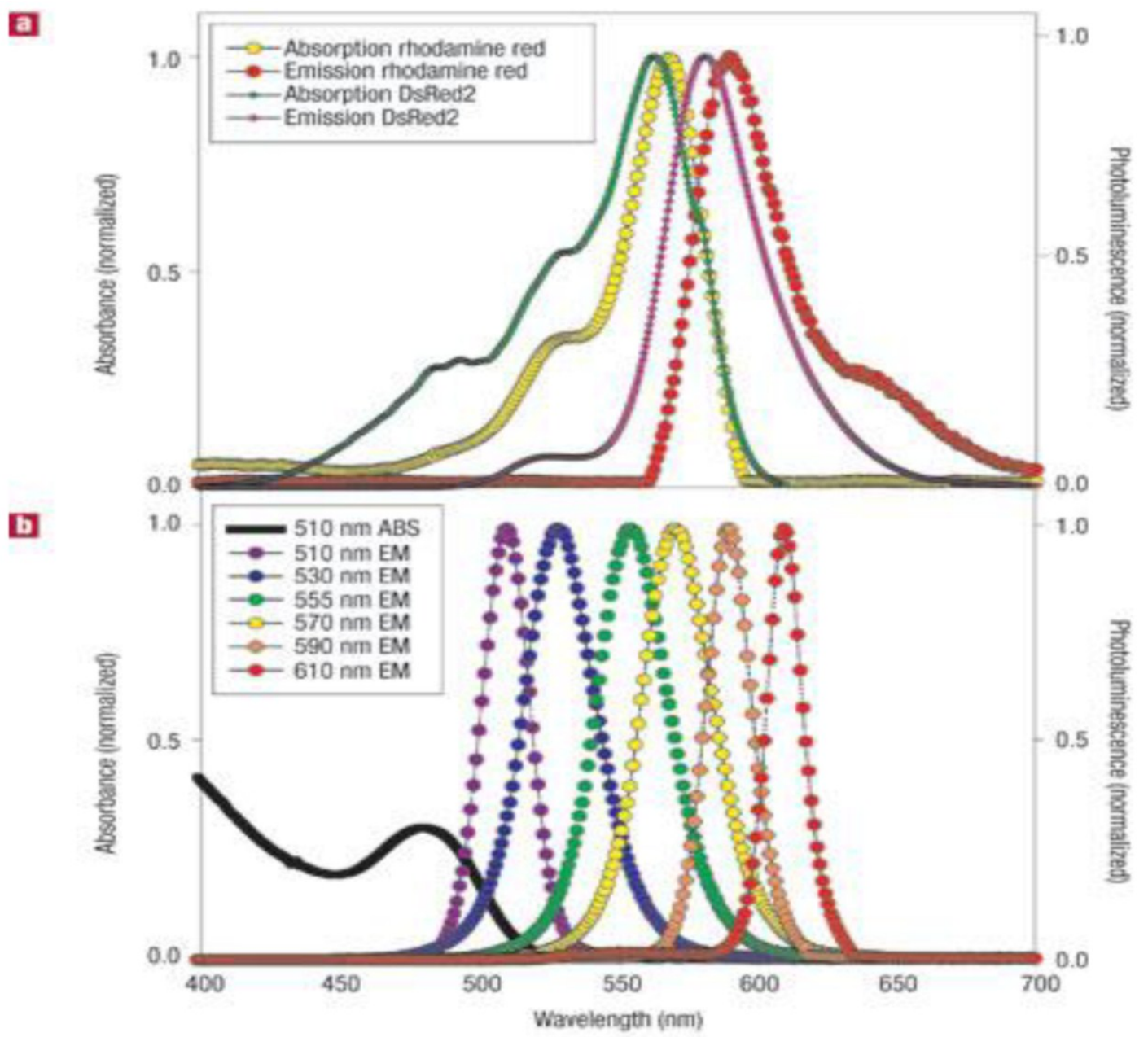

[c

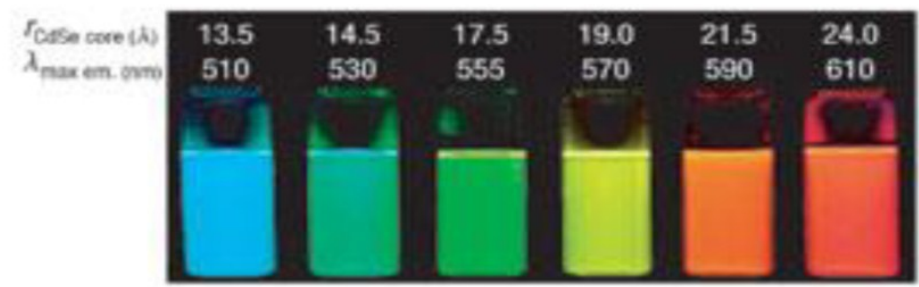

Figure 2. (a) Absorption and emission of rhodamine red, a common organic dye, and genetically-encoded DsRed2 protein. (b) Absorption and emission of different QD dispersions. The black line shows the absorption of the 5I0-nm-emitting QDs. (c) Photo demonstrating the size-tunable luminescence properties and spectral range of the six QD dispersions plotted in b versus CdSe core size. All samples were excited at $365 \mathrm{~nm}$. Reprinted by permission from Macmillan Publishers Ltd: Nature Materials (Medintz IL, Uyeda HT, Goldman ER, Mattoussi H. Quantum dot bioconjugates for imaging, labelling and sensing. Nat Mater. 2005; 4: 435-46., http://www.nature.com), copyright 2005.

\section{Preparation of Bioconjugated Quantum Dots}

High quality core/shell QDs are most often made by using hot colloidal synthesis, which leaves their surface coated with hydrophobic moieties (e.g. trioctylphosphine oxide (TOPO), oleic acid, and/or trioctylphosphine (TOP)), that prohibit their disper- sion in biological fluids. However, water-dispersible QDs with functional groups that facilitate bioconjugation are needed for biological applications [66]. Techniques for transferring hydrophobic core/shell QDs from organic solvents to an aqueous phase have been extensively studied for the last decade. In general, there are two approaches to prepare water-dispersible QDs: (i) functionalizing QD surface with bifunctional molecules such as mercapto acids, 
or hydrophilic dendrimers[67-68]; and (ii) coating the QDs with a biocompatible material such as silica or amphiphilic polymers [69-71]. In the first approach, the preparation of water-dispersible QDs typically involves functionalizing the QD surface with a mercapto acid through ligand exchange [72]. The thiol group of the mercapto acid binds strongly to the QD surface, displacing the existing surface ligands and producing a carboxyl terminated surface suitable for subsequent bioconjugation. For example, hydrophobic QDs can be dispersed into water by replacing the hydrophobic moieties on the QD surface with bidentate dihydrolipoic acid. The second approach involves the encapsulation of QDs with amphiphilic biocompatible polymers[38, 73] or other hydrophilic shell. The encapsulation of QDs with biocompatible polymers allows them to form dispersions in biological buffers that remain stable for months, due to the strong hydrophobic interaction between the hydrophobic moieties on the QD surface and hydrophobic segment of the amphiphile [74]. The overall QD size is much larger when this encapsulation approach is used than when ligand exchange is used. Conjugation of biomolecules to the QD surface remains an important requirement for targeted in vitro and in vivo delivery applications [68]. In general, water-dispersible QDs can be made by terminating their surface with functional groups such as carboxylic acids, primary amines, and thiols. These groups can be linked to targeting ligands using established conjugation chemistry such as carbodiimide, maleimide and succinimide chemistries. Avidin-biotin cross-linking is another popular method for conjugating biomolecules on the surface of QDs. The functionalization and bioconjugation chemistry for QDs is summarized in Figure 3.

\section{Synthesis of Quantum Dots/Drug Formu- lations for Targeted Delivery and Therapy Applications}

QDs provide a versatile platform for engineering traceable drug delivery systems with potential for improving pharmacological treatment of cancers. The mechanism of delivery of QD/drug formulations to tumor cells is determined by the architecture and properties of the nanostructures. Several rules must be considered in preparing QD/drug nanoparticle formulations for targeted therapy in vivo: (i) the nanoparticle surface must be functionalized with targeting ligands for specific delivery to tumor cells and must allow the drug to be delivered together with the carrier; (ii) the size of the nanoparticle must be minimized to allow excretion from the body; (iii) the drug molecules must be confined within the nanoparticle delivery system to prevent any harmful effects to the normal tissue; however, the drug must be released at tumor cells after being triggered externally or by local environmental factors, and (iv) the surface of QDs must be passivated with a long lasting biocompatible polymer to prevent degradation or breakdown of the QDs upon encounter with the biological environment. In the next few paragraphs, we discuss recent findings on the preparation of QD/drug nanoparticle formulations for targeted delivery and therapy.

Two approaches can be used to integrate QDs and drug molecules into a nanoparticle formulation: (i) conjugating or linking drug molecules to the QD surface, followed by delivery of drug-conjugated QDs to specific sites and subsequent release of the drug molecules from the QD surface in response to local biological conditions such as $\mathrm{pH}$ or the presence of enzymes; or (ii) loading the drug in a polymer nanoparticle system that also contains either hydrophobic or hydrophilic QDs, depending on the type of polymer particle used to encapsulate them. The entire $\mathrm{QD} /$ drug nanoparticle system is delivered to the desired organ or tissue, and the drug molecules are either released when the polymer particle is degraded at low $\mathrm{pH}$ or simply diffuse out from polymer particle. For example, Bagalkot et al. employed the first approach, demonstrating the synthesis of QD-aptamer(Apt)-doxorubicin (Dox) conjugates (QD-Apt(Dox)) as a complex conjugate for targeted cancer imaging, therapy, and sensing [75] (see Figure 4). Basically, the QD surface was functionalized with an RNA aptamer that recognizes the extracellular domain of the prostate specific membrane antigen, enabling preferential targeting and imaging of prostate cancer cells. The anticancer drug, Dox, which is intercalated with the RNA aptamer, is released slowly from the QD system. The drug-release process was monitored using the Förster (fluorescence) resonance energy transfer (FRET) between QD and Dox. This system could deliver Dox to the targeted prostate cancer cells and sense the delivery of Dox by activating the fluorescence of QDs that concurrently image the cancer cells. The specificity and sensitivity of this nanoparticle conjugate formulation as a cancer imaging, therapy and sensing system were demonstrated in vitro. Chakravarthy et al. reported on the ability of nanoconjugates of CdSe/CdS/ZnS QD and doxorubicin to target alveolar macrophages, cells that play a critical role in the pathogenesis of inflammatory lung injuries [76]. Confocal imaging showed the release of Dox from the QD-Dox nanoconjugate, as was evident by its accumulation in the cell nucleus and induction of apoptosis, indicating that the drug retains its bio- 
activity after coupling to the nanoparticle. Inflammatory injury parameters (albumin leakage, proinflammatory cytokines, and neutrophil infiltration) were recorded after in vivo administration of QD-Dox and Dox, indicating no significant effect after QD-Dox treatment compared with free Dox. These results show that nanoparticle platforms can provide targeted macrophage-selective therapy for the treatment of pulmonary disease.

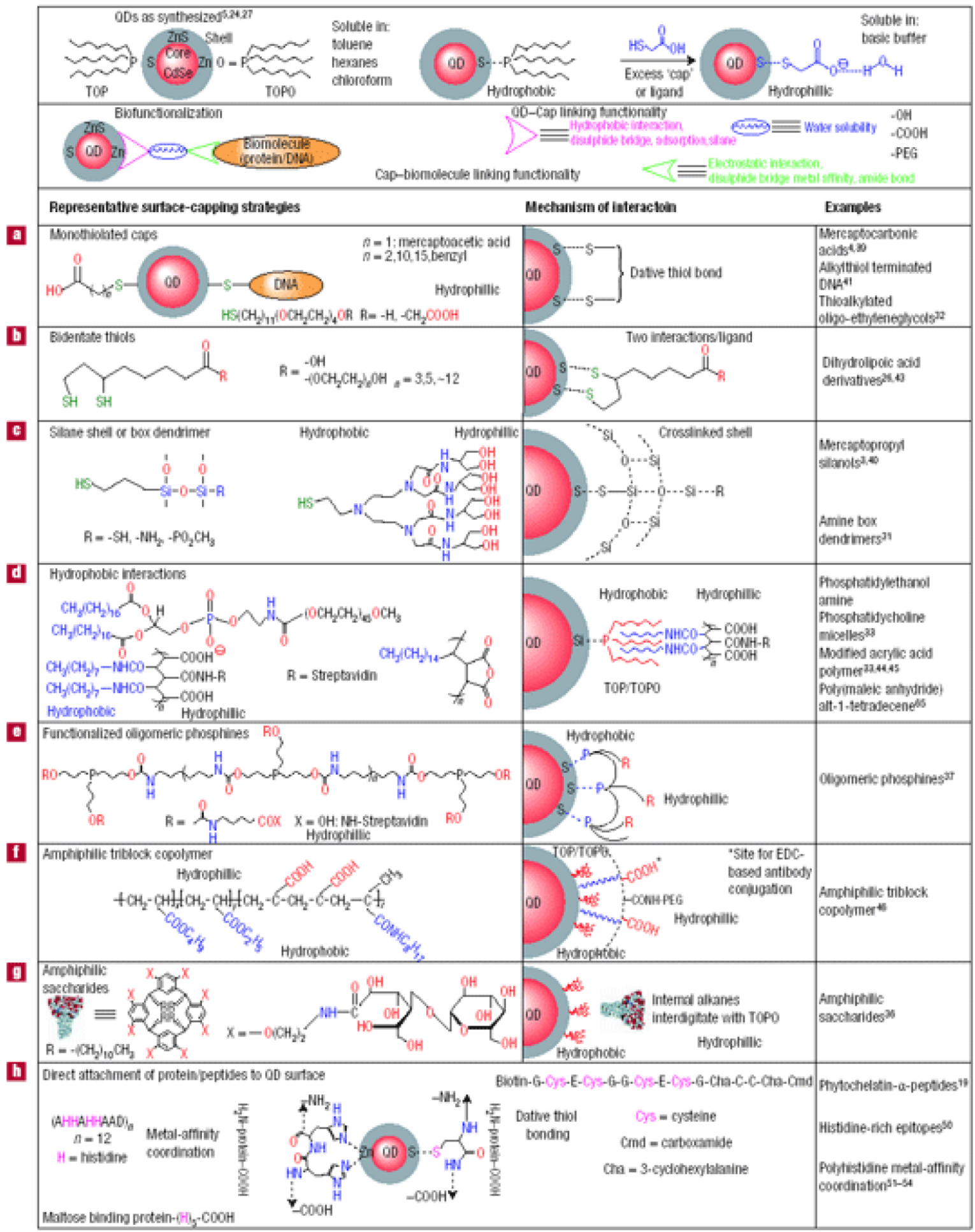

Figure 3. Functionalization and bioconjugation chemistry for QDs. Reprinted by permission from Macmillan Publishers Ltd: Nature Materials (Medintz IL, Uyeda HT, Goldman ER, Mattoussi H. Quantum dot bioconjugates for imaging, labelling and sensing. Nat Mater. 2005; 4: 435-46., http://www.nature.com), copyright 2005. 
(a)

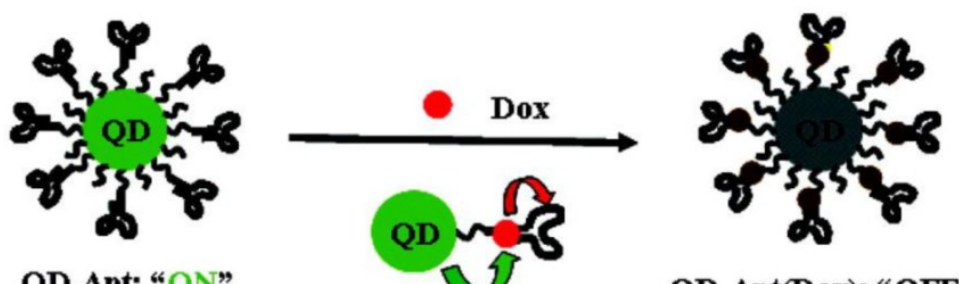

QD-Apt: “ON"

QD-Apt(Dox): “OFF”

(b)

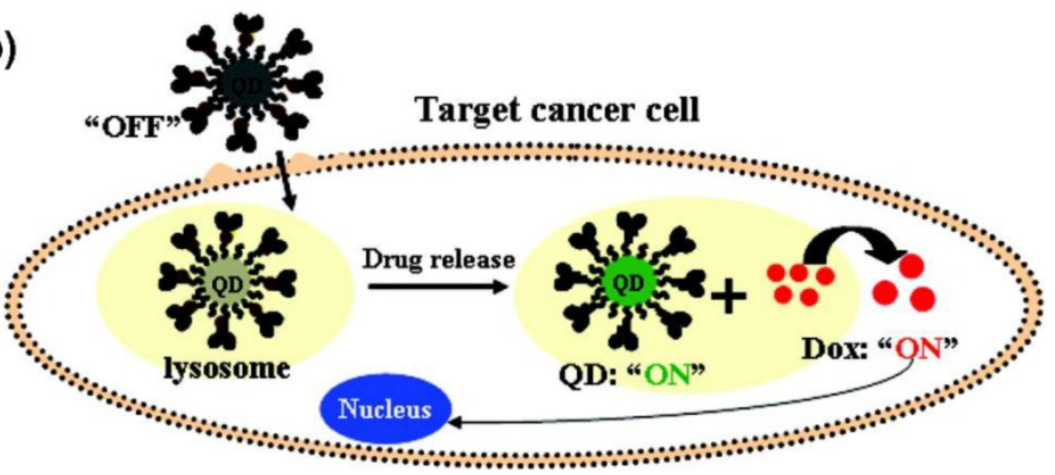

Figure 4. (a) Schematic illustration of QD-Apt(Dox) Bi-FRET system. In the first step, the QD are surface functionalized with the AIO PSMA aptamer. The intercalation of Dox within the AI0 PSMA aptamer on the surface of QDs results in the formation of the QD-Apt(Dox) and quenching of both QD and Dox fluorescence through a Bi-FRET mechanism. (b) Schematic illustration of specific uptake of QD-Apt(Dox) conjugates into target cancer cell through PSMA mediated endocytosis. The release of Dox from the QD-Apt(Dox) conjugates induces the recovery of fluorescence from both QD and Dox, thereby sensing the intracellular delivery of Dox and enabling the synchronous fluorescent localization and killing of cancer cells. Reprinted with permission from (Bagalkot V, Zhang $L$, Levy-Nissenbaum E, Jon S, Kantoff PW, Langer R, et al. Quantum Dotâ^’Aptamer Conjugates for Synchronous Cancer Imaging, Therapy, and Sensing of Drug Delivery Based on Bi-Fluorescence Resonance Energy Transfer. Nano Letters. 2007; 7: 3065-70.). Copyright (2007) American Chemical Society.

Mahajan et al have used an attractive approach where drug delivery has been integrated with site-specificity using a QD-based platform [77]. Specifically, the antiretroviral drug saquinavir and the biorecognition molecule transferrin (Tf) have been conjugated to carboxyl-terminated quantum dots using carbodiimide chemistry. The aim of this study was to significantly enhance the transport of saquinavir into the brain, for the treatment of HIV-1 infected cells within the brain, via targeting the transferrin receptors (TfRs), which are overexpressed on the apical surface of the blood brain barrier (BBB). Using an in vitro model of the $\mathrm{BBB}$, they demonstrated that these targeted and drug-doped QDs can efficiently cross the $\mathrm{BBB}$, and caused a marked decrease in viral replication in the HIV-1 infected peripheral blood mononuclear cells (PBMCs) within the brain. These results highlight the potential of this nanoformulation in the treatment of Neuro-AIDS and other neurological disorders.

Some groups prefer to use the second approach for drug delivery because it does not involve conjugating the drug molecules to the QD surface and purifying the drug-conjugated QDs from the un-reacted drug molecules. Yuan et al. described an approach for combining QD technology with anti-cancer drug therapy [78]. More specifically, blue-emitting $\mathrm{ZnO}$ QDs were combined with biodegradable chitosan for tumor-targeted drug delivery. This showed that the chitosan-coated $\mathrm{ZnO}$ QDs could be loaded with anticancer drug molecules and could deliver anticancer drugs to the tumor. The presence of chitosan on the QDs surface enhanced the colloidal stability of the QDs due to the hydrophilicity and cationic charge of chitosan.

Savla et al. reported the preparation of tumor-targeted, pH-responsive QD-mucin1 aptamer-doxorubicin conjugate for the chemotherapy of ovarian cancer [79]. Basically, the QDs were conjugated with a DNA aptamer specific for mutated MUC1 mucin that is overexpressed in ovarian carcinoma. Doxorubicin was then attached to QD through a $\mathrm{pH}$-sensitive hydrazone bond to provide long-term stability of the complex in systemic circulation and drug release in the acidic environment within tumor cells. The hydrazone bond is stable at neutral and slight basic $\mathrm{pH}$ and undergoes rapid hydrolysis at mildly acidic $\mathrm{pH}$. In vivo studies showed that the QD 
conjugates had higher cytotoxicity than that of the free doxorubicin in cancer cells. More importantly, the QD conjugates were found to be preferably accumulated in the ovarian tumor. The study shows that the proposed QD conjugate has the potential for treating ovarian cancer in vitro and in vivo.

Tian et al. reported the design of drug-loaded liposome-QD hybrid vesicles by incorporating TOPO-coated CdSe/ZnS QDs into two types of lipid bilayers, namely, the 'rigid' disteroylphosphatidylcholine and a fluid-phase bilayer of egg PC.[80] Structural characterization of QD hybrid-vesicles using atomic force microscopy revealed that the QD were incorporated within the lipid membranes of the vesicles. The encapsulation of hydrophilic small molecules (carboxyfluorescein) in the internal aqueous phase of the L-QD hybrids showed different degrees of release in buffer and serum, depending on the type of lipid used. The presence of QD in the lipid bilayer increased the carboxyfluorescein release from the EPC fluid bilayer. In contrast, (DSPC) L-QD hybrids showed a higher stability under the same conditions with minimal carboxyfluorescein leakage. In addition, (DSPC) L-QD hybrids showed no changes in size over a period of three weeks. Finally, doxorubicin (Dox) was loaded into L-QD hybrids using the osmotic gradient technique with at least $97 \%$ loading efficiency. The fluorescence spectrum of Dox was simultaneously detected with that of green-emitting QD that indicated the coexistence of QD and Dox in a single vesicle system. Overall, the drug-loaded L-QD-Dox hybrid vesicles provide a promising multifunctional delivery vector capable of transporting combinations of therapeutic and diagnostic modalities.

$\mathrm{Wu}$ et al. reported polysaccharide-based hybrid nanogels that combine functional building blocks for optical pH-sensing, cancer cell imaging, and controlled drug release within a single nanoparticle system for combined diagnosis and therapy.[81] The hybrid nanogels were synthesized by in-situ immobilization of CdSe QDs in the interior of the dual responsive ( $\mathrm{pH}$ and temperature) hydroxypropylcellulose -poly (acrylic acid) (HPC-PAA) semi-interpenetrating polymer networks. The HPC-PAA-CdSe hybrid nanogels combine a strong trap emission at $741 \mathrm{~nm}$ for sensing physicochemical environment in a $\mathrm{pH}$ dependent manner and a visible excitonic emission at $592 \mathrm{~nm}$ for mouse melanoma B16F10 cell imaging. The hybrid nanogels also provide excellent stability as a drug carrier. They not only provide a high drug loading capacity for a model anticancer drug, temozolomide, but also offer a $\mathrm{pH}$-triggered sustained-release of the drug molecules in the gel network.
Besides the two approaches mentioned above, related approaches have been developed using combinations of both QDs and drug for detecting and treating cancer in vitro. For instance, Mathew et al. demonstrated the fabrication of folic acid-conjugated carboxymethyl chitosan coordinated to Mn-doped ZnS QDs [82]. The system can be used for targeting, controlled drug delivery and imaging of cancer cells. The anticancer drug, 5-Fluorouracil, was incorporated into this QD formulation and was used for the treatment of breast cancer in vitro. The nontoxicity of QD formulation was investigated using L929 cells. Breast cancer cell line MCF-7 was used to study the imaging, targeted delivery and cytotoxicity of the drug-loaded QD formulation. Zhou et al. investigated the use of water-dispersible CdTe QDs coated with negatively charged 3-mercaptopropionic acid to enhance drug uptake into cancer cells. They reported that the MPA-coated CdTe QDs were able to facilitate the interaction of anticancer agent daunorubicin with leukemia cells and enhance the efficiency of biolabeling in cancer cells. Thus, that study demonstrated a potential method for simultaneous cellular inhibition and imaging of cancer cells.

$\mathrm{Li}$ et al. reported an approach to enhance the efficient accumulation of anticancer drug daunorubicin in cancer cells through the combination with CdS QDs [83]. They reported that CdS QDs can readily bind with daunorubicin molecules on the membrane of the cells and promote the uptake of drug molecules in leukemia K562 cells. In addition, the competitive binding of both CdS QDs and anticancer drug to the membrane of leukemia K562 cells could efficiently prevent the drug release by leukemia cells and thus inhibit the possible multidrug resistance of cancer cells, which could be further utilized to improve the drug efficiency in respective tumor chemotherapies in the future.

Apart from the delivery of conventional, small molecule drugs, quantum dots/rods have also been shown to have promise for delivery of more complex biomolecules, such as short interfering RNA (siRNA).[84] These short and double-stranded therapeutic RNA molecules function by blocking the expression of undesirable, disease-causing genes. However, in their free form they have high negative charge and are vulnerable to degradation in physiological fluids. Therefore, for optimal function in vitro and vivo, they must be delivered via electrostatic complexation with cationic nanocariers. Quantum dots/rods, appropriately surface functionalized with cationic moieties, are ideal siRNA carriers as they not only render these genetic drugs with physiological stability and target specificity, but also the whole complex (nanoplex) can 
be optically traced.

We have shown the utility of quantum dot (QD)-siRNA complexes in maintaining the integrity of the blood brain barrier (BBB) (see Figure 5). The matrix-degrading metalloproteinases (MMPs), particularly MMP-9, are involved in neuroinflammation processes leading to disrupting of the BBB, thereby exacerbating neurological diseases such as HIV-1 AIDS dementia and cerebral ischemia.[7] We evaluated the specificity and efficiency of QD complexed with MMP-9-siRNA (nanoplex) in downregulating the expression of MMP-9 in brain microvascular endothelial cells (BMVECs) that constitute the BBB. Silencing MMP-9 gene expression in BMVECs and other cells such as leukocytes may help prevent breakdown of the BBB and inhibit subsequent invasion of the central nervous system (CNS) by infected and inflammatory cells, as well as circulating neurotoxins. Several other groups have also demonstrated the significance of QD/QR mediated delivery of siRNA molecules for the treatment of a variety of ailments, particularly cancer.

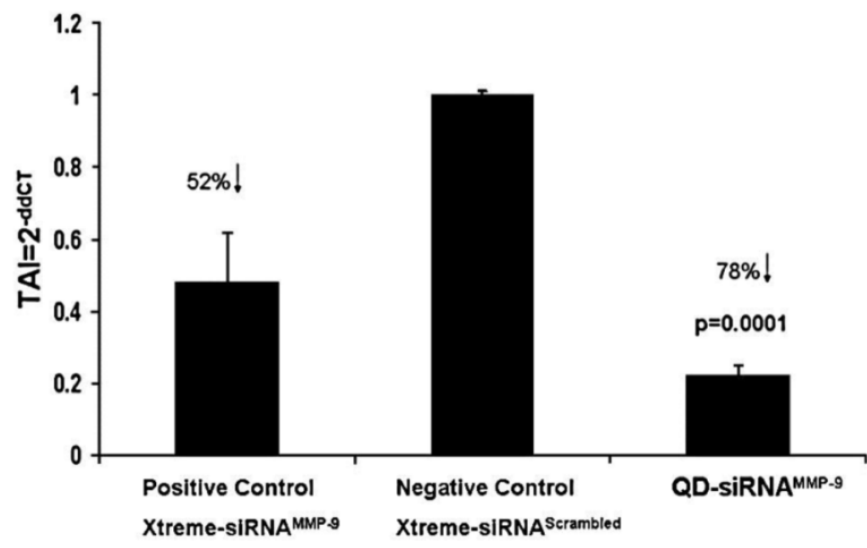

Figure 5. MMP-9 gene silencing in BMVEC by QD-siRNAMMP-9 nanoplexes: BMVECs were transfected with QD-siRNAMMP-9 or Xtreme-siRNAMMP-9 or Xtreme siRNAscrambled for $48 \mathrm{~h}$. RNA was extracted, reverse transcribed, cDNA amplified and MMP-9 gene expression was determined by real-time, quantitative PCR. Relative expression of mRNA species was calculated using the comparative CT method. Data are the mean \pm SD of 3 separate experiments done in duplicate. Statistical significance was determined using ANOVA based comparing QD-siRNAMMP-9 nanoplexes to the negative control samples. Reprinted from Brain Research, 1282, Adela Bonoiu, Supriya D. Mahajan, Ling Ye, Rajiv Kumar, Hong Ding, Ken-Tye Yong, Indrajit Roy, Ravikumar Aalinkeel, Bindukumar Nair, Jessica L. Reynolds, Donald E. Sykes, Marco A. Imperiale, Earl J. Bergey, Stanley A. Schwartz, Paras N. Prasad, MMP-9 gene silencing by a quantum dot-siRNA nanoplex delivery to maintain the integrity of the blood brain barrier, I42-I55, Copyright (2009), with permission from Elsevier.
Finally, it is worth mentioning that QDs have been used as a nanovehicle for the delivery of photosensitizer drugs in photodynamic therapy (PDT), which is a light activated therapy used for the treatment of cancer and other diseases. Here, mainly the drug conjugation approach is used, as unlike in conventional drug delivery, in PDT the release of the drug from its carrier is not a necessity for their therapeutic action. In fact, it has been demonstrated that the efficacy of PDT has been enhanced when the drug is conjugated with the QD system, as a result of favorable energy transfer from the QDs (donor) to the photosensitizer drugs (acceptor). This energy transfer can also facilitate the activation of PDT action at tumor sites located deep within the body. Therefore, QDs can play a pivotal role in enhancing the effectiveness of this newly emerging light activated therapy.

\section{Challenges and opportunities in QD/drug formulation development}

Cytotoxicity is an important factor that must be considered for biological applications [85-87]. The hydrodynamic size, surface charge, dosage, and surface coating of QDs are crucial factors that determine the QD toxicity in vitro and in vivo.[88-89] It is worth noting that the surface coating on the QD surface is not only important for the QDs colloidal stability, but it is essential in preventing the degradation of QDs and the resulting leakage of cadmium ions from the QD core [90]. Moreover, some coating materials have been reported to have toxic effects in cells when the materials are released from the QDs surface. Thus, selecting biocompatible and long lasting polymers to coat the QD surface is essential for long term in vitro and in vivo usage [91]. Oxidation of QD surfaces can occur by multiple processes. Commonly, it happens through radical reactions of oxygen together with UV irradiation [92]. This leads to the formation of chalcogenoxides and release of cadmium ions [93]. The cadmium ions are responsible for the majority of the toxic effects that are ascribed to QDs. It has been suggested that if the surface coating of QDs is maintained during biological applications, the overall cytotoxicity of the QD formulation will decrease.[94] However, if the surface coating is too thick, it may affect the optical and colloidal stability of the QD formulation. Thus, it is important to obtain an optimized formulation where the overall thickness of the surface coating of QDs is near the minimum required to protect the QDs from degradation.

To date, cadmium-based QDs are commonly used for in vitro and in vivo studies [95]. Cadmium and selenium are the main constituent elements in QD 
metalloid complexes. These elements are known to cause acute and chronic toxicities in vertebrates at high dosage. For instance, $\mathrm{Cd}$ ion, a potential carcinogen, can cross the blood-brain barrier (BBB) and placenta, and is systemically distributed to all bodily tissues, with liver and kidney being target organs of toxicity. Recently, it was showed that upon intravenous injection of cadmium-based QDs to small animals, the cadmium concentration in the liver and kidneys increased over the course of four weeks [33]. The cadmium signals in the kidneys reached $10 \%$ of the injected dose as compared to $40 \%$ in the liver. The presence of QDs was observed in both tissue sections of the liver and kidneys using fluorescence microscopy. It was speculated that the redistribution of cadmium over time may result from degradation of QDs in vivo, since the natural accumulation sites of $\mathrm{Cd}$ ions are the liver and kidneys [33]. Therefore, some research groups are currently trying to overcome this challenge by designing cadmium-free QDs to substitute cadmium-based QDs for long term in vivo imaging applications. For example, InP QDs will serve as good candidate for overcoming all these drawbacks as mentioned above. The key advantages offered by InP nanocrystals (as opposed to the cadmium-based QDs which are already commercially available) lies in the robustness of the covalent bond in III-V semiconductors versus the ionic bond in the II-VI semiconductors, as well as the reduced toxicity of compounds such as InP. These QDs can be easily dispersed in aqueous systems using a simple co-ordination reaction with bifunctional ligands such as mercaptoacetic acid (MAA). The extending carboxylic-acid groups on MAA also allows for the attachment of polyethyleneglycol (PEG) and targeting molecules to the QDs and permits specific targeted delivery to tumor cells of interest. To date, comprehensive studies on engineering biocompatible cadmium-free QDs for in vitro and in vivo applications are still rather limited. Thus, there are many opportunities and challenges in the current QDs community that are waiting to be overcome.

\section{Summary and future outlook}

In this review, different types of QD/drug nanoparticle formulations are described for their potential use in targeted delivery and therapy. Using the rich surface functionalization chemistry of QDs, targeting biomolecules and drug formulation can be integrated with QDs for traceable drug delivery and therapy in vitro and in vivo. Many studies have demonstrated that the incorporation of drug formulations with QDs did not compromise the drug efficacy. More importantly, the QD/drug nanoparticle formu- lations were able to serve as an excellent platform for development of a new generation of traceable drug delivery strategies for real time monitoring of the drug biodistribution in vitro and in vivo. Because of toxicity concerns, cadmium-based QDs might not be the best candidate for in vivo drug delivery and therapy. Thus, many research groups are currently synthesizing cadmium-free QDs for in vivo applications. For example, our group has demonstrated the synthesis of indium phosphide, silicon, and copper indium sulfide QDs for targeted tumor imaging and very low toxicity was observed from these formulations[42-43, 96]. However, for in vitro-based drug studies, cadmium-based QDs will remain to be utilized, since toxicity is not a concern. Another potential concern for the use of QDs in delivery and therapy is the overall QD size. In general, it is preferable to minimize the overall size of QDs for in vivo applications to reduce their accumulation in the reticuloendothelial system. Recently, methods have been reported to reduce the size of the QDs by tailoring their surface coating. Finally, passivation of the QD surface with a long-lasting and robust polymer coating is essential to prevent the breakdown of QDs in the biological environment that gives rise to their toxicity. This is a definite concern for in vivo applications. Some reports have suggested that capping the QD core with a higher bandgap semiconductor or biomolecule can minimize the toxicity. But, it is worth noting that each additional step towards functionalizing the QDs will contribute to their final hydrodynamic size and could directly or indirectly affect their biodistribution. In the near future, we envision that the QD/drug nanoparticle formulations will gain wide interest in many healthcare-related research areas. For example, the developed formulations can be used for early cancer detection and therapy. Also, the formulations can be systematically tailored for personalized drug treatment. More importantly, additional modalities such as magnetic resonance imaging and positron emission tomography contrast agents can be integrated into the $\mathrm{QD} /$ drug formulations, thus allowing one to use two or more imaging modalities to verify the biodistribution and efficacy of the drug in vivo. We believe that in the next few years there will be a tremendous growth in developing QD/drug nanoparticle formulations for therapeutic and diagnostic applications.

\section{Acknowledgments}

This study was supported by the Singapore Ministry of Education under Research Grant MOE2010-T2-2-010. 


\section{Competing Interests}

The authors have declared that no competing interest exists.

\section{References}

1. Azzazy HME, Mansour MMH, Kazmierczak SC. From diagnostics to therapy: Prospects of quantum dots. Clinical Biochemistry. 2007; 40: 917-27.

2. Michalet X, Pinaud FF, Bentolila LA, Tsay JM, Doose S, Li JJ, et al. Quantum Dots for Live Cells, in Vivo Imaging, and Diagnostics. Science. 2005; 307: 538-44. doi:10.1126/science.1104274.

3. Prasad PN. Nanophotonics. New York: Wiley-Interscience. 2004.

4. Prasad PN. Biophotonics. New York: Wiley-Interscience. 2004.

5. Farokhzad OC, Langer R. Nanomedicine: Developing smarter therapeutic and diagnostic modalities. Advanced Drug Delivery Reviews. 2006; 58: 1456-9.

6. Harris TJ, Green JJ, Fung PW, Langer R, Anderson DG, Bhatia SN. Tissue-specific gene delivery via nanoparticle coating. Biomaterials. 2010; 31: 998-1006. doi: 10.1016/j.biomaterials.2009.10.012.

7. Bonoiu A, Mahajan SD, Ye L, Kumar R, Ding H, Yong K-T, et al. MMP-9 gene silencing by a quantum dot-siRNA nanoplex delivery to maintain the integrity of the blood brain barrier. Brain Research. 2009; 1282: 142-55. doi: 10.1016/j.brainres.2009.05.047.

8. Shi J, Votruba AR, Farokhzad OC, Langer R. Nanotechnology in Drug Delivery and Tissue Engineering: From Discovery to Applications. Nano Letters. 2010; 10: 3223-30. doi:10.1021/nl102184c.

9. Karnik R, Gu F, Basto P, Cannizzaro C, Dean L, Kyei-Manu W, et al. Microfluidic Platform for Controlled Synthesis of Polymeric Nanoparticles. Nano Letters. 2008; 8: 2906-12. doi:10.1021/nl801736q.

10. Kost J, Langer R. Responsive polymeric delivery systems. Advanced Drug Delivery Reviews. 2001; 46: 125-48. doi: 10.1016/s0169-409x(00)00136-8.

11. Joseph K, Robert L. Responsive polymeric delivery systems. Advanced Drug Delivery Reviews. 2001;6: 19-50. doi: 10.1016/0169-409x(91)90030-g.

12. Alexis F, Rhee J-W, Richie JP, Radovic-Moreno AF, Langer R, Farokhzad OC. New frontiers in nanotechnology for cancer treatment. Urologic Oncology: Seminars and Original Investigations. 2008;26: 74-85. doi: 10.1016/j.urolonc.2007.03.017.

13. Chan JM, Zhang L, Yuet KP, Liao G, Rhee J-W, Langer R, et al. PLGA-lecithin-PEG core-shell nanoparticles for controlled drug delivery. Biomaterials. 2009; 30: 1627-34. doi: 10.1016/j.biomaterials.2008.12.013.

14. Levy-Nissenbaum E, Radovic-Moreno AF, Wang AZ, Langer R, Farokhzad OC. Nanotechnology and aptamers: applications in drug delivery. Trends in Biotechnology. 2008; 26: 442-9. doi: 10.1016/j.tibtech.2008.04.006.

15. Langer R. Transdermal drug delivery: past progress, current status, and future prospects. Advanced Drug Delivery Reviews. 2004; 56: 557-8. doi: 10.1016/j.addr.2003.10.021.

16. Cheng J, Teply BA, Sherifi I, Sung J, Luther G, Gu FX, et al. Formulation of functionalized PLGA-PEG nanoparticles for in vivo targeted drug

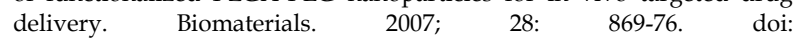
10.1016/j.biomaterials.2006.09.047.

17. Moses MA, Brem H, Langer R. Advancing the field of drug delivery: Taking aim at cancer. Cancer Cell. 2003; 4: 337-41. doi: 10.1016/s1535-6108(03)00276-9.

18. Langer R. Polymer implants for drug delivery in the brain. Journal of Controlled Release. 1991;16: 53-9. doi: 10.1016/0168-3659(91)90030-h.

19. Farokhzad OC, Langer R. Impact of Nanotechnology on Drug Delivery. ACS Nano. 2009; 3: 16-20. doi:10.1021/nn900002m.

20. Valencia PM, Basto PA, Zhang L, Rhee M, Langer R, Farokhzad OC, et al. Single-Step Assembly of Homogenous Lipid-Polymeric and Lipid-Quantum Dot Nanoparticles Enabled by Microfluidic Rapid Mixing. ACS Nano. 2010; 4: 1671-9. doi:10.1021/nn901433u.

21. Bharali DJ, Klejbor I, Stachowiak EK, Dutta P, Roy I, Kaur N, et al. Organically modified silica nanoparticles: A nonviral vector for in vivo gene delivery and expression in the brain. Proceedings of the National Academy of Sciences of the United States of America. 2005; 102: 11539-44. doi:10.1073/pnas.0504926102.

22. Ding H, Yong KT, Roy I, Pudavar HE, Law WC, Bergey EJ, et al. Gold Nanorods Coated with Multilayer Polyelectrolyte as Contrast Agents for Multimodal Imaging. J Phys Chem C. 2007; 111: 12552-7.
23. El-Sayed IH, Huang X, El-Sayed MA. Surface Plasmon Resonance Scattering and Absorption of anti-EGFR Antibody Conjugated Gold Nanoparticles in Cancer Diagnostics: Applications in Oral Cancer. Nano Lett. 2005; 5: 829-34.

24. Ballou B, Ernst LA, Andreko S, Harper T, Fitzpatrick JAJ, Waggoner AS, et al. Sentinel Lymph Node Imaging Using Quantum Dots in Mouse Tumor Models. Bioconjugate Chemistry. 2007; 18: 389-96.

25. Ozkan M. Quantum dots and other nanoparticles: what can they offer to drug discovery? Drug Discovery Today. 2004; 9: 1065-71. doi: 10.1016/s1359-6446(04)03291-x.

26. Shi C, Zhu Y, Cerwinka WH, Zhau HE, Marshall FF, Simons JW, et al. Quantum Dots: Emerging applications in urologic oncology. Urologic Oncology: Seminars and Original Investigations. 2008;26: 86-92. doi: 10.1016/j.urolonc.2007.03.018.

27. Wang C-H, Hsu Y-S, Peng C-A. Quantum dots encapsulated with amphiphilic alginate as bioprobe for fast screening anti-dengue virus agents. Biosensors and Bioelectronics. 2008; 24: 1012-9. doi: 10.1016/j.bios.2008.08.009.

28. Byers RJ, Hitchman ER. Quantum Dots Brighten Biological Imaging. Progress in Histochemistry and Cytochemistry. 2011; 45: 201-37. doi: 10.1016/j.proghi.2010.11.001.

29. Hasegawa U, Nomura S-iM, Kaul SC, Hirano T, Akiyoshi K. Nanogel-quantum dot hybrid nanoparticles for live cell imaging. Biochemical and Biophysical Research Communications. 2005; 331: 917-21. doi: 10.1016/j.bbrc.2005.03.228.

30. Yang K, Cao Y-A, Shi C, Li Z-G, Zhang F-J, Yang J, et al. Quantum dot-based visual in vivo imaging for oral squamous cell carcinoma in mice. Oral Oncology. 2010; 46: 864-8. doi: 10.1016/j.oraloncology.2010.09.009.

31. Yukawa H, Kagami Y, Watanabe M, Oishi K, Miyamoto Y, Okamoto Y, et al. Quantum dots labeling using octa-arginine peptides for imaging of adipose tissue-derived stem cells. Biomaterials. 2010; 31: 4094-103. doi: 10.1016/j.biomaterials.2010.01.134.

32. Juzenas P, Chen W, Sun Y-P, Coelho MAN, Generalov R, Generalova N, et al. Quantum dots and nanoparticles for photodynamic and radiation therapies of cancer. Advanced Drug Delivery Reviews. 2008; 60: 1600-14. doi: 10.1016/j.addr.2008.08.004.

33. Smith AM, Duan H, Mohs AM, Nie S. Bioconjugated quantum dots for in vivo molecular and cellular imaging. Advanced Drug Delivery Reviews. 2008; 60: 1226-40. doi: 10.1016/j.addr.2008.03.015.

34. Praetner M, Rehberg M, Bihari P, Lerchenberger M, Uhl B, Holzer M, et al. The contribution of the capillary endothelium to blood clearance and tissue deposition of anionic quantum dots in vivo. Biomaterials. 2010; 31: 6692-700. doi: 10.1016/j.biomaterials.2010.05.051.

35. Rousserie G, Sukhanova A, Even-Desrumeaux K, Fleury F, Chames P, Baty $\mathrm{D}$, et al. Semiconductor quantum dots for multiplexed bio-detection on solid-state microarrays. Critical Reviews in Oncology/Hematology. 2010; 74: 1-15. doi: 10.1016/j.critrevonc.2009.04.006.

36. Russ Algar W, Krull UJ. Characterization of the adsorption of oligonucleotides on mercaptopropionic acid-coated CdSe/ZnS quantum dots using fluorescence resonance energy transfer. Journal of Colloid and Interface Science. 2011; 359: 148-54. doi: 10.1016/j.jcis.2011.03.058.

37. Bharali DJ, Lucey DW, Jayakumar H, Pudavar HE, Prasad PN. Folate-receptor-mediated delivery of InP quantum dots for bioimaging using confocal and two-photon microscopy. J Am Chem Soc. 2005; 127: 11364-71.

38. Gao X, Cui Y, Levenson RM, Chung LWK, Nie S. In vivo cancer targeting and imaging with semiconductor quantum dots. Nat Biotech. 2004; 22: 969-76.

39. Cai W, Shin DW, Chen K, Gheysens O, Cao Q, Wang SX, et al. Peptide-Labeled Near-Infrared Quantum Dots for Imaging Tumor Vasculature in Living Subjects. Nano Lett. 2006; 6: 669-76.

40. Yong K-T, Hu R, Roy I, Ding H, Vathy LA, Bergey EJ, et al. Tumor Targeting and Imaging in Live Animals with Functionalized Semiconductor Quantum Rods. ACS Applied Materials \& Interfaces. 2009; 1: 710-9. doi:10.1021/am8002318.

41. Yong KT. Biophotonics and Biotechnology in Pancreatic Cancer: Cyclic RGD-Peptide-Conjugated Type II Quantum Dots for in vivo Imaging. Pancreatology. 2010; 10: 553-64.

42. Erogbogbo F, Yong K-T, Roy I, Hu R, Law W-C, Zhao W, et al. In Vivo Targeted Cancer Imaging, Sentinel Lymph Node Mapping and Multi-Channel Imaging with Biocompatible Silicon Nanocrystals. ACS Nano. 2010; 5: 413-23. doi:10.1021/nn1018945.

43. Erogbogbo F, Yong K-T, Hu R, Law W-C, Ding H, Chang C-W, et al. Biocompatible Magnetofluorescent Probes: Luminescent Silicon Quantum Dots Coupled with Superparamagnetic Iron(III) Oxide. ACS Nano. 2010; 4: 5131-8. doi:10.1021/nn101016f. 
44. Adeli M, Hakimpoor F, Parsamanesh M, Kalantari M, Sobhani Z, Attyabi F. Quantum dot-pseudopolyrotaxane supramolecules as anticancer drug delivery systems. Polymer. 2011; doi: 10.1016/j.polymer.2011.02.032.

45. Zrazhevskiy P, Gao X. Multifunctional quantum dots for personalized medicine. Nano Today. 2009; 4: 414-28. doi: 10.1016/j.nantod.2009.07.004.

46. Dorfs D, Krahne R, Falqui A, Manna L, Giannini C, Zanchet D. Quantum Dots: Synthesis and Characterization. In: David LA, Gregory DS, Gary PW, editors. Comprehensive Nanoscience and Technology. Amsterdam: Academic Press. 2011: 219-70.

47. Mokari T, Banin U. Synthesis and Properties of CdSe/ZnS Core/Shell Nanorods. Chem Mater. 2003; 15: 3955-60.

48. Peng ZA, Peng X. Formation of High-Quality CdTe, CdSe, and CdS Nanocrystals Using CdO as Precursor. J Am Chem Soc. 2001; 123: 183-4.

49. Talapin DV, Koeppe R, Gotzinger S, Kornowski A, Lupton JM, Rogach $\mathrm{AL}$, et al. Highly Emissive Colloidal CdSe/CdS Heterostructures of Mixed Dimensionality. Nano Lett. 2003; 3: 1677-81.

50. Rogach AL, Franzl T, Klar TA, Feldmann J, Gaponik N, Lesnyak V, et al. Aqueous Synthesis of Thiol-Capped CdTe Nanocrystals: State-of-the-Art. The Journal of Physical Chemistry C. 2007; 111: 14628-37. doi:10.1021/jp072463y.

51. Murray CB, Norris DJ, Bawendi MG. Synthesis and Characterization of Nearly Monodisperse $\mathrm{CdE}(\mathrm{E}=$ Sulfur, Selenium, Tellurium) Semiconductor Nanocrystallites. J Am Chem Soc. 1993; 115: 8706-15.

52. Murray CB, Kagan CR, Bawendi MG. Self-Organization of CdSe Nanocrystallites into Three-Dimensional Quantum Dot Superlattices. Science. 1995; 270: 1335-8. doi:10.1126/science.270.5240.1335.

53. Peng ZA, Peng X. Nearly Monodisperse and Shape-Controlled CdSe Nanocrystals via Alternative Routes: Nucleation and Growth. J Am Chem Soc. 2002; 124: 3343-53.

54. Dabbousi BO, Rodriguez-Viejo J, Mikulec FV, Heine JR, Mattoussi H, Ober R, et al. (CdSe)ZnS Core-Shell Quantum Dots: Synthesis and Characterization of a Size Series of Highly Luminescent Nanocrystallites. J Phys Chem B. 1997; 101: 9463-75.

55. Peng X, Schlamp MC, Kadavanich AV, Alivisatos AP. Epitaxial Growth of Highly Luminescent CdSe/CdS Core/Shell Nanocrystals with Photostability and Electronic Accessibility. J Am Chem Soc. 1997; 119: 7019-29.

56. Alivisatos $\mathrm{P}$. The use of nanocrystals in biological detection. Nat Biotech. 2004; 22: 47-52.

57. Akerman ME, Chan WCW, Laakkonen P, Bhatia SN, Ruoslahti E. Nanocrystal targeting in vivo. Proceedings of the National Academy of Sciences. 2002; 99: 12617-21. doi:10.1073/pnas.152463399.

58. Bruchez M, Jr., Moronne M, Gin P, Weiss S, Alivisatos AP. Semiconductor Nanocrystals as Fluorescent Biological Labels. Science. 1998; 281: 2013-6. doi:10.1126/science.281.5385.2013.

59. Burda C, Chen X, Narayanan R, El-Sayed MA. Chemistry and Properties of Nanocrystals of Different Shapes. Chem Rev. 2005; 105: 1025-102.

60. Chan WC, et al. Quantum Dot Bioconjugates for Ultrasensitive Nonisotopic Detection. Science. 1998; 281: 2016-8. doi:10.1126/science.281.5385.2016.

61. Courty S, Bouzigues C, Luccardini C, Ehrensperger M, Bonneau S, Dahan $M$, et al. Tracking Individual Proteins in Living Cells Using Single Quantum Dot Imaging. Methods in Enzymology. Academic Press. 2006:211-28.

62. Derfus AM, Chan WCW, Bhatia SN. Intracellular Delivery of Quantum Dots for Live Cell Labeling and Organelle Tracking. Advanced Materials. 2004; 16: 961-6.

63. Costa-Fernández JM, Pereiro R, Sanz-Medel A. The use of luminescent quantum dots for optical sensing. TrAC Trends in Analytical Chemistry. 2006; 25: 207-18.

64. Zimmer JP, Kim SW, Ohnishi S, Tanaka E, Frangioni JV, Bawendi MG. Size Series of Small Indium Arsenide-Zinc Selenide Core-Shell Nanocrystals and Their Application to In Vivo Imaging. J Am Chem Soc. 2006; 128: 2526-7.

65. Jamieson T, Bakhshi R, Petrova D, Pocock R, Imani M, Seifalian AM. Biological applications of quantum dots. Biomaterials. 2007; 28: 4717-32.

66. Dong W, Guo L, Wang M, Xu S. CdTe QDs-based prostate-specific antigen probe for human prostate cancer cell imaging. Journal of Luminescence. 2009; 129: 926-30.

67. Jiang W, Mardyani S, Fischer H, Chan CW. Design and Characterization of Lysine Cross-Linked Mercapto-Acid Biocompatible Quantum Dots. Chem Mater. 2006; 18: 872-8.

68. Larson DR, Zipfel WR, Williams RM, Clark SW, Bruchez MP, Wise FW, et al. Water-Soluble Quantum Dots for Multiphoton Fluorescence Imaging in Vivo. Science. 2003; 300: 1434-6. doi:10.1126/science.1083780.
69. Dubertret B, Skourides P, Norris DJ, Noireaux V, Brivanlou AH, Libchaber A. In Vivo Imaging of Quantum Dots Encapsulated in Phospholipid Micelles. Science. 2002; 298: 1759-62. doi:10.1126/science.1077194.

70. Erogbogbo F, Yong K-T, Roy I, Xu G, Prasad PN, Swihart MT. Biocompatible Luminescent Silicon Quantum Dots for Imaging of Cancer Cells. ACS Nano. 2008; 2: 873-8.

71. Fu A, Gu W, Boussert B, Koski K, Gerion D, Manna L, et al. Semiconductor Quantum Rods as Single Molecule Fluorescent Biological Labels. Nano Lett. 2007; 7: 179-82.

72. Liu W, Choi HS, Zimmer JP, Tanaka E, Frangioni JV, Bawendi M. Compact Cysteine-Coated CdSe(ZnCdS) Quantum Dots for in Vivo Applications. J Am Chem Soc. 2007; 129: 14530-1.

73. Gerion D, Pinaud F, Williams SC, Parak WJ, Zanchet D, Weiss S, et al. Synthesis and Properties of Biocompatible Water-Soluble Silica-Coated CdSe/ZnS Semiconductor Quantum Dots. J Phys Chem B. 2001; 105: 8861-71.

74. Duan H, Nie S. Cell-Penetrating Quantum Dots Based on Multivalent and Endosome-Disrupting Surface Coatings. Journal of the American Chemical Society. 2007; 129: 3333-8.

75. Bagalkot V, Zhang L, Levy-Nissenbaum E, Jon S, Kantoff PW, Langer R, et al. Quantum Dotâ^Aptamer Conjugates for Synchronous Cancer Imaging, Therapy, and Sensing of Drug Delivery Based on Bi-Fluorescence Resonance Energy Transfer. Nano Letters. 2007; 7: 3065-70.

76. Chakravarthy KV, Davidson BA, Helinski JD, Ding H, Law W-C, Yong $\mathrm{K}-\mathrm{T}$, et al. Doxorubicin-conjugated quantum dots to target alveolar macrophages and inflammation. Nanomedicine: Nanotechnology, Biology and Medicine. 2011; 7: 88-96. doi: 10.1016/j.nano.2010.09.001.

77. Mahajan SD, Roy I, Xu G, Yong K-T, Ding H, Aalinkeel R, et al. Enhancing the Delivery of Anti Retroviral Drug "Saquinavir" Across the Blood Brain Barrier Using Nanoparticles. Current HIV Research. 2010; 9: 396-404.

78. Yuan Q, Hein S, Misra RDK. New generation of chitosan-encapsulated $\mathrm{ZnO}$ quantum dots loaded with drug: Synthesis, characterization and in vitro drug delivery response. Acta Biomaterialia. 2010; 6: 2732-9. doi: 10.1016/j.actbio.2010.01.025.

79. Savla R, Taratula O, Garbuzenko O, Minko T. Tumor targeted quantum dot-mucin 1 aptamer-doxorubicin conjugate for imaging and treatment of cancer. Journal of Controlled Release. 2011; doi: 10.1016/j.jconrel.2011.02.015

80. Tian B, Al-Jamal WT, Al-Jamal KT, Kostarelos K. Doxorubicin-loaded lipid-quantum dot hybrids: Surface topography and release properties. International Journal of Pharmaceutics. 2011;doi: 10.1016/j.ijpharm.2011.01.057.

81. Wu W, Aiello M, Zhou T, Berliner A, Banerjee P, Zhou S. In-situ immobilization of quantum dots in polysaccharide-based nanogels for integration of optical $\mathrm{pH}$-sensing, tumor cell imaging, and drug delivery. Biomaterials. 2010; 31: 3023-31. doi: 10.1016/j.biomaterials.2010.01.011.

82. Mathew ME, Mohan JC, Manzoor K, Nair SV, Tamura H, Jayakumar R. Folate conjugated carboxymethyl chitosan-manganese doped zinc sulphide nanoparticles for targeted drug delivery and imaging of cancer cells. Carbohydrate Polymers. 2010; 80: 442-8. doi: 10.1016/j.carbpol.2009.11.047.

83. Li J, Wu C, Gao F, Zhang R, Lv G, Fu D, et al. In vitro study of drug accumulation in cancer cells via specific association with CdS nanoparticles. Bioorganic \& Medicinal Chemistry Letters. 2006; 16: 4808-12. doi: 10.1016/j.bmcl.2006.06.069.

84. Xu G, Yong K-T, Roy I, Mahajan SD, Ding H, Schwartz SA, et al. Bioconjugated Quantum Rods as Targeted Probes for Efficient Transmigration Across an in Vitro Blood\&\#x2212;Brain Barrier. Bioconjugate Chem. 2008; 19: 1179-85.

85. Chen Z, Chen H, Meng H, Xing G, Gao X, Sun B, et al. Bio-distribution and metabolic paths of silica coated CdSeS quantum dots. Toxicology and Applied Pharmacology. 2008; 230: 364-71. doi: 10.1016/j.taap.2008.03.022.

86. Li KG, Chen JT, Bai SS, Wen X, Song SY, Yu Q, et al. Intracellular oxidative stress and cadmium ions release induce cytotoxicity of unmodified cadmium sulfide quantum dots. Toxicology in Vitro. 2009; 23: 1007-13. doi: 10.1016/j.tiv.2009.06.020.

87. Rzigalinski BA, Strobl JS. Cadmium-containing nanoparticles: Perspectives on pharmacology and toxicology of quantum dots. Toxicology and Applied Pharmacology. 2009; 238: 280-8. doi: 10.1016/j.taap.2009.04.010.

88. Zhang LW, Yu WW, Colvin VL, Monteiro-Riviere NA. Biological interactions of quantum dot nanoparticles in skin and in human 
epidermal keratinocytes. Toxicology and Applied Pharmacology. 2008; 228: 200-11. doi: 10.1016/j.taap.2007.12.022.

89. Zhao Y, Lin K, Zhang W, Liu L. Quantum dots enhance Cu2+-induced hepatic L02 cells toxicity. Journal of Environmental Sciences. 2010; 22: 1987-92. doi: 10.1016/s1001-0742(09)60350-8.

90. Hezinger AFE, Teßmar J, Göpferich A. Polymer coating of quantum dots - A powerful tool toward diagnostics and sensorics. European Journal of Pharmaceutics and Biopharmaceutics. 2008; 68: 138-52. doi: 10.1016/j.ejpb.2007.05.013.

91. Edmund AR, Kambalapally S, Wilson TA, Nicolosi RJ. Encapsulation of cadmium selenide quantum dots using a self-assembling nanoemulsion (SANE) reduces their in vitro toxicity. Toxicology in Vitro. 2011; 25: 185-90. doi: 10.1016/j.tiv.2010.10.017.

92. Cho SJ, Maysinger D, Jain M, Roder B, Hackbarth S, Winnik FM. Long-Term Exposure to CdTe Quantum Dots Causes Functional Impairments in Live Cells. Langmuir. 2007; 23: 1974-80.

93. Su Y, He Y, Lu H, Sai L, Li Q, Li W, et al. The cytotoxicity of cadmium based, aqueous phase - Synthesized, quantum dots and its modulation by surface coating. Biomaterials. 2009; 30: 19-25.

94. Derfus AM, Chan WCW, Bhatia SN. Probing the Cytotoxicity of Semiconductor Quantum Dots. Nano Lett. 2004; 4: 11-8.

95. Yong K-T, Roy I, Swihart MT, Prasad PN. Multifunctional nanoparticles as biocompatible targeted probes for human cancer diagnosis and therapy. Journal of Materials Chemistry. 2009; 19: 4655-72.

96. Yong K-T, Ding H, Roy I, Law W-C, Bergey EJ, Maitra A, et al. Imaging Pancreatic Cancer Using Bioconjugated InP Quantum Dots. ACS Nano. 2009; 3: 502-10. doi:10.1021/nn8008933. 